\title{
REGULARITY OF WEAK SOLUTIONS AND SUPERSOLUTIONS TO THE POROUS MEDIUM EQUATION
}

\author{
VERENA BÖGELEIN, PEKKA LEHTELÄ, AND STEFAN STURM
}

\begin{abstract}
We study the relations between different regularity assumptions in the definition of weak solutions and supersolutions to the porous medium equation. In particular, we establish the equivalence of the conditions $u^{m} \in L_{\mathrm{loc}}^{2}\left(0, T ; H_{\mathrm{loc}}^{1}(\Omega)\right)$ and $u^{\frac{m+1}{2}} \in L_{\mathrm{loc}}^{2}\left(0, T ; H_{\mathrm{loc}}^{1}(\Omega)\right)$ in the definition of weak solutions. Our proof is based on approximation by solutions to obstacle problems.
\end{abstract}

\section{INTRODUCTION}

In this paper, we study the connections between various notions of nonnegative (super)solutions to the slow diffusion porous medium equation

$$
\partial_{t} u-\Delta u^{m}=0 \text { in } \Omega_{T},
$$

where $m>1$ and $\Omega_{T}=\Omega \times(0, T)$ with $T>0$ denotes the space-time cylinder over a bounded domain $\Omega \subset \mathbb{R}^{n}$. Particularly, concerning the notion of weak solution in the case $m>1$, there are basically two different definitions used in the literature. The difference becomes apparent in the regularity assumptions utilized in the definition of weak solutions. The first one, which is used for instance in $[5,11,17,21]$, acts on the assumptions that

$$
u \in C^{0}\left((0, T) ; L_{\mathrm{loc}}^{m+1}(\Omega)\right) \text { and } u^{m} \in L_{\mathrm{loc}}^{2}\left(0, T ; H_{\mathrm{loc}}^{1}(\Omega)\right),
$$

whereas the requirements in the other definition of weak solutions, used for instance in $[4,12,13,14]$, are

$$
u \in C^{0}\left((0, T) ; L_{\mathrm{loc}}^{2}(\Omega)\right) \quad \text { and } \quad u^{\frac{m+1}{2}} \in L_{\mathrm{loc}}^{2}\left(0, T ; H_{\mathrm{loc}}^{1}(\Omega)\right) .
$$

As the relations between these conditions have, at least to the authors' knowledge, not been treated up to now, we aim to clarify this matter by establishing the equivalence of these two conditions in the definition of weak solutions; see Theorem 1.2 below.

As a tool, which is interesting also in its own right, we consider a class of supersolutions that are defined analogously to supercaloric functions in classical potential theory, i. e. in terms of the parabolic comparison principle. Originally, this class was introduced as "viscosity supersolutions" in [17], and recently, the

\footnotetext{
Date: September 20, 2018.

2010 Mathematics Subject Classification. Primary 35D10, Secondary 35K65, 35K10.

Key words and phrases. Porous medium equation, weak solutions, weak supersolutions, gradient estimates, obstacle problem.

The research is partially supported by the Emil Aaltonen Foundation.
} 
label " $m$-superporous functions" was suggested in $[18,19]$. As it seems to be more natural, we will call them m-supercaloric functions (see Def. 2.2). Apart from that, another notion is the one of weak supersolutions (see Def. 2.1), which are defined via the weak formulation of (1.1). While $m$-supercaloric functions typically appear in potential theory, the weak formulation provides a natural approach to regularity questions.

In [17], it was proved that these two notions are related in the sense that bounded $m$-supercaloric functions are also weak supersolutions to the porous medium equation. According to that result, $m$-supercaloric functions can be studied via approximation by their truncations, which are weak supersolutions. However, the defintion of weak supersolutions used for example in $[15,17]$ does not allow to work with the solution itself as a test function. Instead, only $u^{m}$ is an admissible choice. Therefore, some additional assumptions are needed when testing the equation with $u$. A sufficient condition, that is commonly imposed (see $[4,12,13,14]$ ), is given by the integrability property (1.2).

Our first theorem shows that this property is satisfied for bounded $\mathrm{m}$ supercaloric functions. In a sense, it complements the work of [17] as it allows to study the regularity of $m$-supercaloric functions by applying the results for weak supersolutions which were established under the assumption (1.2).

Theorem 1.1. Let $u$ be a locally bounded $m$-supercaloric function. Then, $u$ is a weak supersolution to the porous medium equation (1.1) and u satisfies

$$
u^{\frac{m+1}{2}} \in L_{\mathrm{loc}}^{2}\left(0, T ; H_{\mathrm{loc}}^{1}(\Omega)\right) \text {. }
$$

The novelty of this paper is proving the existence of the gradient $\nabla u^{\frac{m+1}{2}}$ in $L_{\text {loc }}^{2}\left(\Omega_{T}, \mathbb{R}^{n}\right)$ for locally bounded $m$-supercaloric functions $u$. Moreover, we rigorously establish all the tools concerning solutions to the obstacle problem used throughout the proof, like the assertion that weak solutions to the obstacle problem are weak solutions to the porous medium equation in the complement of the coincidence set (see Lemma 4.2). This closes a gap in the literature, since such assertions were commonly recognized to be true although to our knowledge, a rigorous proof was missing.

From the above theorem, we can also infer the equivalence of locally bounded $m$-supercaloric functions and weak supersolutions satisfying (1.2) in the following way. First, our theorem shows that locally bounded $m$-supercaloric functions are weak supersolutions fulfilling the conditions $u^{m} \in L_{\mathrm{loc}}^{2}\left(0, T ; H_{\mathrm{loc}}^{1}(\Omega)\right)$ and $u^{\frac{m+1}{2}} \in L_{\text {loc }}^{2}\left(0, T ; H_{\text {loc }}^{1}(\Omega)\right)$. On the other hand, the fact that weak supersolutions are $m$-supercaloric functions follows directly from the comparison principle (see [21, Thm. 6.5]) and the existence of lower semicontinuous representatives (see [2, Thm. 1.1]).

The analogue result for the evolutionary $p$-Laplace equation was established in [16], where the counterpart of $m$-supercaloric functions are $p$-superparabolic functions. In other words, there was shown that bounded $p$-superparabolic functions are weak supersolutions to the evolutionary $p$-Laplace equation, and the other implication follows in the same manner as for the porous medium 
equation. However, despite the similarities of both equations, the necessity of a condition like (1.2) is typical for the porous medium equation whereas such a phenomenon does not appear in the theory of the $p$-Laplace equation.

Coming back to the porous medium equation, similar challenges as for weak supersolutions also arise when trying to define a suitable notion of weak solution in order to establish their regularity. However, as weak solutions possess a continuous representative by [9, Thm. 1.1], we can assume that they are locally bounded (see also [1] for an explicit estimate), and since also the comparison principle is at hand, Theorem 1.1 is applicable. This shows that, for weak solutions, $u^{m} \in L_{\mathrm{loc}}^{2}\left(0, T ; H_{\mathrm{loc}}^{1}(\Omega)\right)$ implies $u^{\frac{m+1}{2}} \in L_{\mathrm{loc}}^{2}\left(0, T ; H_{\mathrm{loc}}^{1}(\Omega)\right)$. On the one hand, this result can be seen as a direct consequence of Theorem 1.1. On the other hand, it can be proved directly, without employing the theory of $m$ supercaloric functions. Since also this viewpoint is interesting - in particular for more general porous medium type equations - we provide this alternative proof at the end of Section 5. In addition, we will even prove the converse statement, which ensures that we will get the following equivalence of the two notions of weak solutions to the porous medium equation mentioned above.

Theorem 1.2. For any function $u: \Omega_{T} \rightarrow[0, \infty]$, the following statements are equivalent:

(i) $u \in C^{0}\left((0, T) ; L_{\mathrm{loc}}^{m+1}(\Omega)\right)$ and $u^{m} \in L_{\mathrm{loc}}^{2}\left(0, T ; H_{\mathrm{loc}}^{1}(\Omega)\right)$ and

$$
\iint_{\Omega_{T}}\left(-u \partial_{t} \varphi+\nabla u^{m} \cdot \nabla \varphi\right) d x d t=0
$$

for any test function $\varphi \in C_{0}^{\infty}\left(\Omega_{T}\right)$;

(ii) $u \in C^{0}\left((0, T) ; L_{\mathrm{loc}}^{2}(\Omega)\right)$ and $u^{\frac{m+1}{2}} \in L_{\mathrm{loc}}^{2}\left(0, T ; H_{\mathrm{loc}}^{1}(\Omega)\right)$ and

$$
\iint_{\Omega_{T}}\left(-u \partial_{t} \varphi+\frac{2 m}{m+1} u^{\frac{m-1}{2}} \nabla u^{\frac{m+1}{2}} \cdot \nabla \varphi\right) d x d t=0
$$

for any test function $\varphi \in C_{0}^{\infty}\left(\Omega_{T}\right)$.

Remark 1.3. The assertion (i) provides the standard weak formulation coming from multiplication by a test function and integration by parts in (1.1). By contrast, under the assumption $u^{\frac{m+1}{2}} \in L_{\text {loc }}^{2}\left(0, T ; H_{\text {loc }}^{1}(\Omega)\right)$, the weak formulation of (1.1) can be defined as in (ii) if we understand the gradient $\nabla u^{\frac{m+1}{2}}$ in the sense

$$
\nabla u^{\frac{m+1}{2}}=\frac{m+1}{2 m} \chi_{\{u>0\}} u^{\frac{1-m}{2}} \nabla u^{m} .
$$

Note that this interpretation is in accordance with the definition of weak solutions given, for instance, in [4, Def. 1.1] and [15, eq. (5.7)].

To conclude the introduction, we will present the basic ideas of the proof of Theorem 1.1, which will imply Theorem 1.2 as described. Since an $m$ supercaloric function $u$ is lower semicontinuous, it can be approximated by an increasing sequence of smooth functions $\psi_{i}$. The idea is to consider solutions $u_{i}$ to the obstacle problem with $\psi_{i}$ as an obstacle. Then, it can be 
shown that $u_{i}$ are weak supersolutions and $u_{i} \rightarrow u$ in a suitable sense. Our contribution here is providing uniform $L_{\mathrm{loc}}^{2}\left(\Omega_{T}, \mathbb{R}^{n}\right)$-estimates for the gradients $\nabla u_{i}^{\frac{m+1}{2}}$ of the weak solutions to the obstacle problem, which ensure that $\nabla u^{\frac{m+1}{2}} \in L_{\mathrm{loc}}^{2}\left(\Omega_{T}, \mathbb{R}^{n}\right)$ after passing to the limit $i \rightarrow \infty$. Moreover, as mentioned before, we provide rigorous proofs for some tools on obstacle problems which were commonly recognized to be true, like the assertion on the complement of the coincidence set in Lemma 4.2. In a certain sense, this complements the work of [7]. More precisely, in Sections 3 and 4, we modify their approach of constructing weak solutions to the obstacle problem by establishing the estimates for $\nabla u_{i}^{\frac{m+1}{2}}$ which were not taken into account in [7]. Finally, via the described estimates and weak compactness, we can conclude that (1.2) is valid.

\section{Preliminaries}

To start with, we fix some notations. We let $\Omega$ be a bounded domain in $\mathbb{R}^{n}$ and denote by $\Omega_{T}:=\Omega \times(0, T)$ the space-time cylinder of height $T>0$ over $\Omega$. For $U \Subset \Omega$ and $0<t_{1}<t_{2}<T$, we abbreviate the cylinder $U \times\left(t_{1}, t_{2}\right)$ by $U_{t_{1}, t_{2}}$ and its parabolic boundary by $\partial_{p} U_{t_{1}, t_{2}}:=\left(\partial U \times\left(t_{1}, t_{2}\right)\right) \cup\left(\bar{U} \times\left\{t_{1}\right\}\right)$. For an open ball of radius $\varrho>0$ centered at $x_{0} \in \Omega$, we write $B\left(x_{0}, \varrho\right)$. Moreover, we denote the positive and negative parts of a function $u$ by $u_{+}:=\max \{u, 0\}$ and $u_{-}:=\max \{-u, 0\}$, respectively, and, $C$ stands for a constant, which may vary from line to line.

Next, we define our notion of weak (super)solutions.

Definition 2.1. A non-negative $u \in C^{0}\left((0, T) ; L_{\mathrm{loc}}^{m+1}(\Omega)\right)$ is a weak supersolution to the porous medium equation (1.1) if $u^{m} \in L_{\mathrm{loc}}^{2}\left(0, T ; H_{\mathrm{loc}}^{1}(\Omega)\right)$ and $u$ satisfies

$$
\iint_{\Omega_{T}}\left(-u \partial_{t} \varphi+\nabla u^{m} \cdot \nabla \varphi\right) d x d t \geq 0
$$

for any test function $\varphi \in C_{0}^{\infty}\left(\Omega_{T}\right)$ with $\varphi \geq 0$. Similarly, $u$ is a weak subsolution if the above inequality holds reversed. Moreover, $u$ is a weak solution if it is a weak sub- and supersolution.

We continue by giving the definition of $m$-supercaloric functions.

Definition 2.2. A function $u: \Omega_{T} \rightarrow[0, \infty]$ is m-supercaloric if

(1) $u$ is lower semicontinuous,

(2) $u$ is finite in a dense subset of $\Omega_{T}$, and

(3) $u$ satisfies the following comparison principle in every interior cylinder $U_{t_{1}, t_{2}} \Subset \Omega_{T}$ : If $w \in C^{0}\left(\overline{U_{t_{1}, t_{2}}}\right)$ is a weak solution to (1.1) in $U_{t_{1}, t_{2}}$ and $u \geq w$ on $\partial_{p} U_{t_{1}, t_{2}}$, then $u \geq w$ in $U_{t_{1}, t_{2}}$.

By [17, Thm. 1.3], bounded $m$-supercaloric functions are weak supersolutions in the sense of Def. 2.1.

In order to derive energy estimates for weak (super)solutions to the porous medium equation, we want to use the (super)solution $u$ itself as a test function. However, since the time derivative $\partial_{t} u$ does not exist in general, we 
need to regularize $u$ to deal with this matter. To that end, we introduce the mollification

$$
\llbracket u \rrbracket_{h}(x, t)=e^{-\frac{t}{h}} v_{0}+\frac{1}{h} \int_{0}^{t} e^{\frac{s-t}{h}} u(x, s) d s
$$

for $h>0$ with some $v_{0} \in L^{1}(\Omega)$. Then, choosing $v_{0} \equiv 0$, it can be shown that $\llbracket u \rrbracket_{h}$ satisfies the regularized inequality (see [7, eq. (6.5)] or [17, eq. (2.12)])

$$
\iint_{\Omega_{T}}\left(\partial_{t} \llbracket u \rrbracket_{h} \varphi+\nabla \llbracket u^{m} \rrbracket_{h} \cdot \nabla \varphi\right) d x d t \geq \frac{1}{h} \int_{\Omega} u(\cdot, 0) \int_{0}^{T} \varphi e^{-\frac{s}{h}} d s d x
$$

for any test function $\varphi \in L^{2}\left(0, T ; H_{0}^{1}(\Omega)\right)$. Moreover, the formula

$$
\partial_{t} \llbracket u \rrbracket_{h}=\frac{1}{h}\left(u-\llbracket u \rrbracket_{h}\right)
$$

holds (see [7, Lemma 3.1]). By imposing the additional regularity condition (1.2) to a weak supersolution $u$, we can now derive the following Caccioppolitype estimate.

Lemma 2.3. Let $u$ be a weak supersolution to the porous medium equation in the sense of Def. 2.1 which additionally satisfies $u^{\frac{m+1}{2}} \in L_{\mathrm{loc}}^{2}\left(0, T ; H_{\mathrm{loc}}^{1}(\Omega)\right)$, and let $\zeta \in C_{0}^{\infty}\left(\Omega_{T}\right)$ be a smooth cut-off function such that $0 \leq \zeta \leq 1$. Then, if $u \leq M$ in $\Omega_{T}$ for some constant $M>0$, the Caccioppoli estimate

$$
\begin{aligned}
& \iint_{\Omega_{T}} \zeta^{2}\left|\nabla u^{\frac{m+1}{2}}\right|^{2} d x d t \\
& \quad \leq C\left(\iint_{\Omega_{T}}(M-u)^{2} \zeta\left|\partial_{t} \zeta\right| d x d t+\iint_{\Omega_{T}} u^{m-1}(M-u)^{2}|\nabla \zeta|^{2} d x d t\right)
\end{aligned}
$$

holds with a constant $C=C(m)$.

Proof. For $\varepsilon \in(0, M)$, we let $g_{\varepsilon}(s):=M-\max \{\varepsilon, s\}$ for any $s \geq 0$. Moreover, we recall Definition (2.2) of the mollification in time, where throughout this proof we choose $v_{0}=0$ as initial value. In the regularized inequality (2.3), we insert the test function $\varphi=\zeta^{2} g_{\varepsilon}(u)$. Note that $\varphi$ is admissible since $g_{\varepsilon}(u) \in L_{\text {loc }}^{2}\left(0, T ; W_{\text {loc }}^{1,2}(\Omega)\right)$. We first treat the evolutionary integral. Using formula (2.4) for the time derivative of the mollification, integrating by parts and then passing in turn to the limits $h \rightarrow 0$ and $\varepsilon \rightarrow 0$, we find

$$
\begin{aligned}
& \iint_{\Omega_{T}} \zeta^{2} \partial_{t} \llbracket u \rrbracket_{h} g_{\varepsilon}(u) d x d t \\
& \quad=\iint_{\Omega_{T}} \zeta^{2} \partial_{t} \llbracket u \rrbracket_{h} g_{\varepsilon}\left(\llbracket u \rrbracket_{h}\right) d x d t+\iint_{\Omega_{T}} \zeta^{2} \partial_{t} \llbracket u \rrbracket_{h}\left(g_{\varepsilon}(u)-g_{\varepsilon}\left(\llbracket u \rrbracket_{h}\right)\right) d x d t \\
& \quad \leq \iint_{\Omega_{T}} \zeta^{2} \partial_{t}\left(\int_{M}^{\llbracket u \rrbracket_{h}} g_{\varepsilon}(s) d s\right) d x d t=-2 \iint_{\Omega_{T}} \zeta \partial_{t} \zeta \int_{M}^{\llbracket u \rrbracket_{h}} g_{\varepsilon}(s) d s d x d t \\
& \quad \rightarrow-2 \iint_{\Omega_{T}} \zeta \partial_{t} \zeta \int_{M}^{u}(M-s) d s d x d t=\iint_{\Omega_{T}} \zeta \partial_{t} \zeta(M-u)^{2} d x d t .
\end{aligned}
$$


Note that, in the limit $h \rightarrow 0$, the right-hand side term in (2.3) vanishes, and the diffusion term reads as

$$
\begin{aligned}
& 2 \iint_{\Omega_{T}} \zeta g_{\varepsilon}(u) \nabla u^{m} \cdot \nabla \zeta d x d t-\iint_{\Omega_{T} \cap\{u>\varepsilon\}} \zeta^{2} \nabla u^{m} \cdot \nabla u d x d t \\
& =\frac{4 m}{m+1} \iint_{\Omega_{T}} \zeta g_{\varepsilon}(u) u^{\frac{m-1}{2}} \nabla u^{\frac{m+1}{2}} \cdot \nabla \zeta d x d t-\frac{4 m}{(m+1)^{2}} \iint_{\Omega_{T} \cap\{u>\varepsilon\}} \zeta^{2}\left|\nabla u^{\frac{m+1}{2}}\right|^{2} d x d t .
\end{aligned}
$$

Finally, after letting $\varepsilon \rightarrow 0$, an application of Young's inequality shows that this expression can be bounded from above by

$$
2 m \iint_{\Omega_{T}} u^{m-1}(M-u)^{2}|\nabla \zeta|^{2} d x d t-\frac{2 m}{(m+1)^{2}} \iint_{\Omega_{T}} \zeta^{2}\left|\nabla u^{\frac{m+1}{2}}\right|^{2} d x d t,
$$

which proves the claim.

As the proof of Theorem 1.1 is based on approximations by solutions to obstacle problems for the porous medium equation, we introduce the concept of obstacle problems now. The idea is to find a function $u$ lying above a given obstacle function $\psi$ and attaining fixed boundary and initial values $g$ and $u_{0}$. In addition to that, $u$ needs to fulfill a variational inequality. More precisely, formally $u$ is required to satisfy

$$
\left\{\begin{array}{l}
\iint_{\Omega_{T}}\left[\partial_{t} u\left(v^{m}-u^{m}\right)+\nabla u^{m} \cdot\left(\nabla v^{m}-\nabla u^{m}\right)\right] d x d t \geq 0 \\
u \geq \psi \text { a. e. in } \Omega_{T} \\
u=g \text { on } \partial \Omega \times(0, T) \\
u(\cdot, 0)=u_{0} \text { in } \Omega
\end{array}\right.
$$

for all comparison maps $v \geq \psi$ which take the same boundary and initial values as $u$. For our purposes, it suffices to consider boundary and initial values given by the obstacle function $\psi$. However, the following estimates hold also in the case of sufficiently regular data $g$ and $u_{0}$ as long as they are bounded.

We consider two classes of solutions to the obstacle problem, namely strong and weak solutions (see Def. 2.4 and Def. 2.5). Since we are interested in approximating bounded $m$-supercaloric functions by solutions to obstacle problems, it is reasonable to assume that the obstacles $\psi$ are smooth and bounded, which guarantees the existence of weak solutions to the obstacle problem. If, in addition, the function $\partial_{t} \psi-\Delta \psi^{m}$ is bounded, we have strong solutions to the obstacle problem. The main difference between both notions is that $\partial_{t} u$ exists in a distributional sense only for strong solutions.

The reader should be aware that the proof of Theorem 1.1 has to be performed locally as in [17, Thm. 3.2], meaning that we need to work in a cylinder which is compactly contained in $\Omega_{T}$. However, for the sake of a friendly notation, in Sections 2-4, we will work in the whole domain $\Omega_{T}$, having in mind that the explicit argumentation in Section 5 will be done in a smaller cylinder.

Before we give the rigorous definition of strong solutions, we specify the regularity assumptions for the obstacle and the boundary and initial values. 
First, we want all data to be non-negative and bounded in the sense that

$$
\psi, g \in L^{\infty}\left(\Omega_{T}\right) \text { and } u_{0} \in L^{\infty}(\Omega) .
$$

Next, we impose the compatibility conditions

$$
\left\{\begin{array}{l}
g \geq \psi \text { a. e. in } \Omega_{T}, \\
u_{0} \geq \psi(\cdot, 0) \text { a.e. in } \Omega, \\
g(\cdot, 0)=u_{0} \text { a.e. in } \Omega,
\end{array}\right.
$$

and the following integrability assumptions

$$
\left\{\begin{array}{l}
\psi^{m} \in L^{2}\left(0, T ; H^{1}(\Omega)\right), \partial_{t} \psi^{m} \in L^{\frac{m+1}{m}}\left(\Omega_{T}\right), \psi^{m}(\cdot, 0) \in H^{1}(\Omega), \\
g^{m} \in L^{2}\left(0, T ; H^{1}(\Omega)\right), \partial_{t} g^{m} \in L^{\frac{m+1}{m}}\left(\Omega_{T}\right), \\
u_{0} \in H^{1}(\Omega) .
\end{array}\right.
$$

Note that the assumptions (2.8) on $\psi$ and $g$ imply $\psi^{m}, g^{m} \in$ $C^{0}\left([0, T] ; L^{\frac{m+1}{m}}(\Omega)\right)$, and consequently $\psi, g \in C^{0}\left([0, T] ; L^{m+1}(\Omega)\right)$ since $m>1$. Finally, in order to prove the existence of strong solutions, we need the extra condition

$$
\Psi:=\partial_{t} \psi-\Delta \psi^{m} \in L^{\infty}\left(\Omega_{T}\right) .
$$

Definition 2.4. Let (2.6)-(2.9) be satisfied. A non-negative function $u \in$ $C^{0}\left([0, T] ; L^{m+1}(\Omega)\right)$ is a strong solution to the obstacle problem $(2.5)$ with boundary and initial values $g$ and $u_{0}$ if $u$ fulfills

$$
\left\{\begin{array}{l}
u^{m} \in g^{m}+L^{2}\left(0, T ; H_{0}^{1}(\Omega)\right) \\
u \geq \psi \text { a. e. in } \Omega_{T} \\
\partial_{t} u \in L^{2}\left(0, T ; H^{-1}(\Omega)\right)
\end{array}\right.
$$

$u(\cdot, 0)=u_{0}$ in the $H^{-1}(\Omega)$-sense and the inequality

$$
\int_{0}^{T}\left\langle\partial_{t} u, \alpha\left(v^{m}-u^{m}\right)\right\rangle d t+\iint_{\Omega_{T}} \alpha \nabla u^{m} \cdot \nabla\left(v^{m}-u^{m}\right) d x d t \geq 0
$$

for all comparison maps $v$ satisfying the conditions (2.10) and for all nonnegative cut-off functions $\alpha \in W^{1, \infty}([0, T])$ with $\alpha(T)=0$, where $\langle\cdot, \cdot\rangle$ denotes the dual pairing between $H^{-1}(\Omega)$ and $H_{0}^{1}(\Omega)$.

However, the smoothness and boundedness of $\psi$ are not enough to guarantee that (2.9) holds. Thus, we also need to consider weak solutions to the obstacle problem. We remark that even though (2.6) will be satisfied throughout this paper, it is not a necessary assumption to ensure the existence of weak solutions.

Definition 2.5. Let (2.7) and (2.8) be satisfied. A non-negative function $u \in C^{0}\left([0, T] ; L^{m+1}(\Omega)\right)$ is a weak solution to the obstacle problem (2.5) with boundary and initial values $g$ and $u_{0}$ if $u$ fulfills

$$
\left\{\begin{array}{l}
u^{m} \in g^{m}+L^{2}\left(0, T ; H_{0}^{1}(\Omega)\right) \\
u \geq \psi \text { a. e. in } \Omega_{T}
\end{array}\right.
$$


and the inequality

$$
\left\langle\left\langle\partial_{t} u, \alpha\left(v^{m}-u^{m}\right)\right\rangle\right\rangle_{u_{0}}+\iint_{\Omega_{T}} \alpha \nabla u^{m} \cdot \nabla\left(v^{m}-u^{m}\right) d x d t \geq 0
$$

for all comparison maps $v$ satisfying the conditions (2.12) and $\partial_{t} v^{m} \in$ $L^{\frac{m+1}{m}}\left(\Omega_{T}\right)$, and for all non-negative cut-off functions $\alpha \in W^{1, \infty}([0, T])$ with $\alpha(T)=0$, where we have denoted

$$
\begin{aligned}
\left\langle\left\langle\partial_{t} u, \alpha\left(v^{m}-u^{m}\right)\right\rangle\right\rangle_{u_{0}}: & =\iint_{\Omega_{T}}\left(\alpha^{\prime}\left(\frac{1}{m+1} u^{m+1}-u v^{m}\right)-\alpha u \partial_{t} v^{m}\right) d x d t \\
& +\alpha(0) \int_{\Omega}\left(\frac{1}{m+1} u_{0}^{m+1}-u_{0} v^{m}(\cdot, 0)\right) d x .
\end{aligned}
$$

Note that the initial condition $u(\cdot, 0)=u_{0}$ is incorporated in the variational inequality (2.13); see [7, Lemma 5.2].

Remark 2.6. The strong and weak solutions to the obstacle problem constructed in Sections 3 and 4 are also weak supersolutions to the porous medium equation (see [7, Thms. 2.6, 2.7]). Thus, energy estimates for weak supersolutions such as Lemma 2.3 hold, provided that the regularity assumption (1.2) is satisfied.

Finally, we cite the following parabolic Sobolev's inequality from [10, Prop. I.3.1] (see also [4, Lemma 2.1]).

Lemma 2.7. Let $B\left(x_{0}, \varrho\right) \subset \Omega$ and $0<t_{1}<t_{2}<T$. If

$$
v \in L^{\infty}\left(t_{1}, t_{2} ; L^{r}\left(B\left(x_{0}, \varrho\right)\right)\right) \cap L^{p}\left(t_{1}, t_{2} ; W^{1, p}\left(B\left(x_{0}, \varrho\right)\right)\right)
$$

with $p \in(1, \infty)$ and $r \in[1, \infty)$, there exists a constant $C=C(n, p, r)$ such that

$$
\begin{aligned}
& \int_{t_{1}}^{t_{2}} \int_{B\left(x_{0}, \varrho\right)}|v|^{\ell} d x d t \\
& \quad \leq C \int_{t_{1}}^{t_{2}} \int_{B\left(x_{0}, \varrho\right)}\left(\left|\frac{v}{\varrho}\right|^{p}+|\nabla v|^{p}\right) d x d t\left(\operatorname{ess~sup~}_{t \in\left(t_{1}, t_{2}\right)} \int_{B\left(x_{0}, \varrho\right)}|v|^{r} d x\right)^{p / n},
\end{aligned}
$$

where $\ell=p \frac{n+r}{n}$.

\section{Gradient estimates for Strong solutions}

In this section, we deal with strong solutions to the obstacle problem and assume that (2.6)-(2.9) hold. We will show the existence of strong solutions to the obstacle problem by approximating them by weak solutions to the penalized porous medium equation

$$
\left\{\begin{array}{l}
\partial_{t} u-\Delta u^{m}=\Psi_{+} \xi_{\delta}\left(\psi^{m}-u^{m}\right) \text { in } \Omega_{T} \\
u=g \text { on } \partial \Omega \times(0, T) \\
u(\cdot, 0)=u_{0} \text { in } \Omega .
\end{array}\right.
$$


Here, for $\delta>0$, the function $\xi_{\delta}: \mathbb{R} \rightarrow[0,1]$ is smooth and satisfies

$$
\left\{\begin{array}{l}
\xi_{\delta}=1 \quad \text { in }[0, \infty), \\
\xi_{\delta}=0 \quad \text { in }(-\infty,-\delta], \\
\left|\xi_{\delta}^{\prime}\right| \leq \frac{2}{\delta}
\end{array}\right.
$$

Observe that, whenever $u^{m} \geq \psi^{m}+\delta$, the term $\xi_{\delta}\left(\psi^{m}-u^{m}\right)$ vanishes and thus, (3.1) reduces to the ordinary initial-boundary value problem for the porous medium equation. Next, we give the rigorous definition for weak solutions to the above penalized equation.

Definition 3.1. A non-negative function $u \in C^{0}\left([0, T] ; L^{m+1}(\Omega)\right)$ is a weak solution to the penalized porous medium equation (3.1) if $u$ fulfills

$$
\left\{\begin{array}{l}
u^{m} \in g^{m}+L^{2}\left(0, T ; H_{0}^{1}(\Omega)\right) \\
u(\cdot, 0)=u_{0} \text { in } \Omega
\end{array}\right.
$$

and the equation

$$
\iint_{\Omega_{T}}\left(-u \partial_{t} \varphi+\nabla u^{m} \cdot \nabla \varphi\right) d x d t=\iint_{\Omega_{T}} \Psi_{+} \xi_{\delta}\left(\psi^{m}-u^{m}\right) \varphi d x d t
$$

for any test function $\varphi \in C_{0}^{\infty}\left(\Omega_{T}\right)$.

The associated averaged equation can be deduced as in [7, eq. (6.5)] and is given by

$$
\begin{aligned}
& \iint_{\Omega_{T}}\left(\partial_{t} \llbracket u \rrbracket_{h} \varphi+\nabla \llbracket u^{m} \rrbracket_{h} \cdot \nabla \varphi\right) d x d t \\
& \quad=\iint_{\Omega_{T}} \llbracket \xi_{\delta}\left(\psi^{m}-u^{m}\right) \Psi_{+} \rrbracket_{h} \varphi d x d t+\frac{1}{h} \int_{\Omega} u_{0} \int_{0}^{T} \varphi e^{-\frac{s}{h}} d s d x
\end{aligned}
$$

for any test function $\varphi \in L^{2}\left(0, T ; H_{0}^{1}(\Omega)\right)$. As before, $\llbracket \cdot \rrbracket_{h}$ is defined according to $(2.2)$ with $v_{0}=0$. We cite the following energy estimates for weak solutions to the penalized porous medium equation from [7, Lemma 7.2].

Lemma 3.2. Let $u$ be a weak solution to (3.1). Then, we have

$$
\sup _{t \in[0, T]} \int_{\Omega} u(\cdot, t)^{m+1} d x+\iint_{\Omega_{T}}\left|\nabla u^{m}\right|^{2} d x d t \leq C A
$$

and

$$
\left\|\partial_{t} u\right\|_{L^{2}\left(0, T ; H^{-1}(\Omega)\right)}^{2} \leq C A .
$$

Here, $C$ is a constant depending on $n, m, \operatorname{diam}(\Omega)$ and $T$, and $A$ is defined as

$$
\begin{aligned}
A= & \sup _{t \in[0, T]} \int_{\Omega} g(\cdot, t)^{m+1} d x+\int_{\Omega} u_{0}^{m+1} d x \\
& +\iint_{\Omega_{T}}\left(\left|\Psi_{+}\right|^{2}+\left|\nabla g^{m}\right|^{2}+\left|\partial_{t} g^{m}\right|^{\frac{m+1}{m}}\right) d x d t .
\end{aligned}
$$


The proof follows from a formal calculation by inserting the test function $\varphi=\chi_{[0, \tau]}\left(u^{m}-g^{m}\right)$ for some $\tau \in(0, T]$ in $(3.3)$, where $\chi_{[0, \tau]}$ denotes the characteristic function of the interval $[0, \tau]$. For the details, we refer to [7].

Next, we will prove the following gradient estimate.

Lemma 3.3. Let $u$ be a weak solution to the penalized porous medium equation (3.1) and suppose that $u \in L_{\mathrm{loc}}^{2}\left(0, T ; H_{\mathrm{loc}}^{1}(\Omega)\right)$. Then, we have $\nabla u^{\frac{m+1}{2}} \in$ $L_{\mathrm{loc}}^{2}\left(\Omega_{T}, \mathbb{R}^{n}\right)$, together with the estimate

$$
\iint_{U_{t_{1}, t_{2}}}\left|\nabla u^{\frac{m+1}{2}}\right|^{2} d x d t \leq C(A+1)
$$

for any $U \Subset \Omega$ and any $0<t_{1}<t_{2}<T$ with $A$ as in (3.4) and a constant $C=C\left(n, m, U, t_{1}, t_{2}, \Omega, T\right)$.

Proof. We fix $U, t_{1}, t_{2}$ as in the statement of the lemma. To show that $\nabla u^{\frac{m+1}{2}}$ exists, we define $u_{k}=\min \{u, k\}$ for $k \in \mathbb{N}$, and note that $[0, k] \ni s \mapsto s^{\frac{m+1}{2}}$ is Lipschitz continuous so that $u_{k}^{\frac{m+1}{2}}$ is weakly differentiable with

$$
\nabla u_{k}^{\frac{m+1}{2}}=\frac{m+1}{2} u_{k}^{\frac{m-1}{2}} \nabla u_{k}
$$

More precisely, for any $i \in\{1, \ldots, n\}$, we have that

$$
\iint_{U_{t_{1}, t_{2}}} u_{k}^{\frac{m+1}{2}} \partial_{x_{i}} \varphi d x d t=-\frac{m+1}{2} \iint_{U_{t_{1}, t_{2}}} u_{k}^{\frac{m-1}{2}} \partial_{x_{i}} u_{k} \varphi d x d t
$$

for any $\varphi \in C_{0}^{\infty}\left(U_{t_{1}, t_{2}}\right)$. As the right-hand side remains bounded in the limit $k \rightarrow \infty$, the gradient $\nabla u^{\frac{m+1}{2}}$ exists and is given by $\nabla u^{\frac{m+1}{2}}=\frac{m+1}{2} u^{\frac{m-1}{2}} \nabla u$. Next, in order to prove the gradient bound, we choose a non-negative cut-off function $\zeta \in C_{0}^{\infty}\left(\Omega_{T}\right)$ with $\zeta=1$ in $U_{t_{1}, t_{2}}$ and $|\nabla \zeta|+\left|\partial_{t} \zeta\right| \leq \frac{C}{\operatorname{dist}\left(U_{t_{1}, t_{2}}, \partial \Omega_{T}\right)}$. Then, we insert the test function $\varphi=\zeta^{2} u$ in the regularized equation (3.3). We begin by treating the term involving the time derivative. Observing that, by $(2.4)$, we have

$$
\begin{aligned}
\iint_{\Omega_{T}} \zeta^{2} u \partial_{t} \llbracket u \rrbracket_{h} d x d t & =\iint_{\Omega_{T}} \zeta^{2} \llbracket u \rrbracket_{h} \partial_{t} \llbracket u \rrbracket_{h} d x d t+\iint_{\Omega_{T}} \zeta^{2}\left(u-\llbracket u \rrbracket_{h}\right) \partial_{t} \llbracket u \rrbracket_{h} d x d t \\
& \geq \frac{1}{2} \iint_{\Omega_{T}} \zeta^{2} \partial_{t} \llbracket u \rrbracket_{h}^{2} d x d t=-\iint_{\Omega_{T}} \zeta \partial_{t} \zeta \llbracket u \rrbracket_{h}^{2} d x d t
\end{aligned}
$$

and considering [16, Lemma 2.2] for the convergence properties of the mollification, we may let $h \rightarrow 0$. Then, inserting the above inequality in (3.3), we get

$$
\iint_{\Omega_{T}}\left(-\zeta \partial_{t} \zeta u^{2}+\nabla u^{m} \cdot \nabla\left(\zeta^{2} u\right)\right) d x d t \leq \iint_{\Omega_{T}} \zeta^{2} u \Psi_{+} \xi_{\delta}\left(\psi^{m}-u^{m}\right) d x d t .
$$

By Young's inequality, we may write

$$
\begin{aligned}
\nabla u^{m} \cdot \nabla\left(\zeta^{2} u\right) & =\frac{4 m}{(m+1)^{2}} \zeta^{2}\left|\nabla u^{\frac{m+1}{2}}\right|^{2}+\frac{4 m}{m+1} \zeta u^{\frac{m+1}{2}} \nabla u^{\frac{m+1}{2}} \cdot \nabla \zeta \\
& \geq \frac{2 m}{(m+1)^{2}} \zeta^{2}\left|\nabla u^{\frac{m+1}{2}}\right|^{2}-2 m u^{m+1}|\nabla \zeta|^{2} .
\end{aligned}
$$


Furthermore, we treat the penalty term by applying Young's inequality to $u \Psi_{+}$ and by using the facts that $\zeta \leq 1$ and $\xi_{\delta} \leq 1$. In this way, we find

$$
\begin{aligned}
& \iint_{\Omega_{T}} \zeta^{2}\left|\nabla u^{\frac{m+1}{2}}\right|^{2} d x d t \\
& \quad \leq C \iint_{\Omega_{T}}\left(u^{m+1}|\nabla \zeta|^{2}+\Psi_{+}^{2}+u^{2}+\zeta\left|\partial_{t} \zeta\right| u^{2}\right) d x d t \\
& \quad \leq C \iint_{\Omega_{T}}\left(u^{m+1}|\nabla \zeta|^{2}+\Psi_{+}^{2}+u^{m+1}+\left(\zeta\left|\partial_{t} \zeta\right|\right)^{\frac{m+1}{m-1}}+1\right) d x d t
\end{aligned}
$$

and, employing the $L^{\infty}\left(0, T ; L^{m+1}(\Omega)\right)$-bound for $u$ from Lemma 3.2, this inequality proves the claim.

Now, we will show that there exists a weak solution to (3.1) satisfying the above energy estimates. Our contribution is proving that the estimate for $\nabla u^{\frac{m+1}{2}}$ holds, and for the reader's convenience, we present the key ideas of the existence proof from [7, Lemma 7.3] as well.

Lemma 3.4. There exists a weak solution $u_{\delta}$ to (3.1) such that $u_{\delta} \geq \psi$ a.e. in $\Omega_{T}$. Moreover, we have $\nabla u_{\delta}^{\frac{m+1}{2}} \in L_{\text {loc }}^{2}\left(\Omega_{T}, \mathbb{R}^{n}\right)$, and the following estimates hold:

$$
\sup _{t \in[0, T]} \int_{\Omega} u_{\delta}^{m+1} d x+\left\|\nabla u_{\delta}^{m}\right\|_{L^{2}\left(\Omega_{T}, \mathbb{R}^{n}\right)}+\left\|\partial_{t} u_{\delta}\right\|_{L^{2}\left(0, T ; H^{-1}(\Omega)\right)} \leq C_{1}
$$

and

$$
\left\|\nabla u_{\delta}^{\frac{m+1}{2}}\right\|_{L^{2}\left(U_{\left.t_{1}, t_{2}, \mathbb{R}^{n}\right)}\right.} \leq C_{2},
$$

where $C_{1}=C_{1}(n, m, \Omega, T, A)$ and $C_{2}=C_{2}\left(n, m, U, t_{1}, t_{2}, \Omega, T, A\right)$ are constants and $A$ is as in (3.4).

Proof. For $\varepsilon, \gamma, \delta \in(0,1]$, we define

$$
\begin{aligned}
& \psi_{\varepsilon}=\psi+\varepsilon, \\
& g_{\varepsilon, \gamma}=\left(g^{m}+\gamma^{m}\right)^{\frac{1}{m}}+\varepsilon, \\
& u_{0, \varepsilon, \gamma}=u_{0}+\varepsilon+\gamma, \\
& \Psi_{\varepsilon}=\partial_{t} \psi_{\varepsilon}-\Delta \psi_{\varepsilon}^{m} .
\end{aligned}
$$

One can easily see that $g_{\varepsilon, \gamma} \geq g+\varepsilon \geq \psi_{\varepsilon}$ and $u_{0, \varepsilon, \gamma} \geq \psi_{\varepsilon}(\cdot, 0)$. Denoting

$$
N=\max \left\{\sup _{\Omega_{T}}\left(\psi_{\varepsilon}^{m}+\delta\right)^{\frac{1}{m}}, \sup _{\Omega_{T}} g_{\varepsilon, \gamma}, \sup _{\Omega} u_{0, \varepsilon, \gamma}\right\}
$$

it follows that $\psi_{\varepsilon}, g_{\varepsilon, \gamma}, u_{0, \varepsilon, \gamma} \leq N$, and by choosing $\varepsilon$ and $\gamma$ smaller if necessary, we can assure that $N \leq \frac{1}{\varepsilon+\gamma}$. Next, we define

$$
a_{\varepsilon}(s)= \begin{cases}m \varepsilon^{m-1}, & 0 \leq s \leq \varepsilon, \\ m s^{m-1}, & \varepsilon<s \leq \frac{1}{\varepsilon}, \\ m \varepsilon^{1-m}, & s \geq \frac{1}{\varepsilon}\end{cases}
$$


Then, by [20, Thm. 1.2 , p. 162 f.], there exists a weak solution $u_{\varepsilon, \gamma, \delta} \in$ $C^{0}\left([0, T] ; L^{2}(\Omega)\right) \cap L^{2}\left(0, T ; H^{1}(\Omega)\right)$ to the initial-boundary value problem

$$
\left\{\begin{array}{l}
\partial_{t} u-\operatorname{div}\left(a_{\varepsilon}(u) \nabla u\right)=\left(\Psi_{\varepsilon}\right)_{+} \xi_{\delta}\left(\psi_{\varepsilon}^{m}-u^{m}\right) \text { in } \Omega_{T}, \\
u=g_{\varepsilon, \gamma} \text { on } \partial \Omega \times(0, T), \\
u(\cdot, 0)=u_{0, \varepsilon, \gamma} \text { in } \Omega .
\end{array}\right.
$$

Since $u_{\varepsilon, \gamma, \delta}$ satisfies the comparison principle (see [7, Lemma 7.1]) and the constants $\varepsilon+\gamma$ and $N$ are solutions, we have

$$
\varepsilon+\gamma \leq u_{\varepsilon, \gamma, \delta} \leq N \leq \frac{1}{\varepsilon+\gamma} \quad \text { a. e. in } \Omega_{T} \text {. }
$$

Thus, we obtain $a_{\varepsilon}\left(u_{\varepsilon, \gamma, \delta}\right) \nabla u_{\varepsilon, \gamma, \delta}=\nabla u_{\varepsilon, \gamma, \delta}^{m}$, which implies that $u_{\varepsilon, \gamma, \delta}$ is also a weak solution to (3.1) with boundary values $g_{\varepsilon, \gamma}$ and initial values $u_{0, \varepsilon, \gamma}$. Hence, by Lemma 3.2 and Lemma 3.3, we have the following energy estimates for $u_{\varepsilon, \gamma, \delta}$ :

$$
\sup _{t \in[0, T]} \int_{\Omega} u_{\varepsilon, \gamma, \delta}^{m+1} d x+\left\|\nabla u_{\varepsilon, \gamma, \delta}^{m}\right\|_{L^{2}\left(\Omega_{T}, \mathbb{R}^{n}\right)}+\left\|\partial_{t} u_{\varepsilon, \gamma, \delta}\right\|_{L^{2}\left(0, T ; H^{-1}(\Omega)\right)} \leq C_{1}
$$

and

$$
\left\|\nabla u_{\varepsilon, \gamma, \delta}^{\frac{m+1}{2}}\right\|_{L^{2}\left(U_{\left.t_{1}, t_{2}, \mathbb{R}^{n}\right)}\right.} \leq C_{2}
$$

for any $U \Subset \Omega$ and any $0<t_{1}<t_{2}<T$ with constants $C_{1}=C_{1}(n, m, \Omega, T, A)$ and $C_{2}=C_{2}\left(n, m, U, t_{1}, t_{2}, \Omega, T, A\right)$, where $A$ is as in (3.4). Note that $C_{1}$ and $C_{2}$ are independent of $\varepsilon, \gamma$ and $\delta$. Moreover, by [11, Thm. 1.2], the weak solutions $u_{\varepsilon, \gamma, \delta}$ are locally Hölder continuous with an estimate independent of $\varepsilon$. Therefore, we may let $\varepsilon \rightarrow 0$ and, subsequently by monotone convergence, $\gamma \rightarrow 0$ to conclude that $u_{\varepsilon, \gamma, \delta}$ subconverge to a weak solution $u_{\delta}$ to the penalized porous medium equation in the following sense: $u_{\varepsilon, \gamma, \delta} \rightarrow u_{\delta}$ a. e. in $\Omega_{T}, \nabla u_{\varepsilon, \gamma, \delta}^{m} \rightarrow \nabla u_{\delta}^{m}$ weakly in $L^{2}\left(\Omega_{T}, \mathbb{R}^{n}\right), u_{\varepsilon, \gamma, \delta} \stackrel{*}{\rightarrow} u_{\delta}$ weakly-* in $L^{\infty}\left(0, T ; L^{m+1}(\Omega)\right), \partial_{t} u_{\varepsilon, \gamma, \delta} \rightarrow \partial_{t} u_{\delta}$ weakly in $L^{2}\left(0, T ; H^{-1}(\Omega)\right)$, and $\nabla u_{\varepsilon, \gamma, \delta}^{\frac{m+1}{2}} \rightarrow \nabla u_{\delta}^{\frac{m+1}{2}}$ weakly in $L^{2}\left(U_{t_{1}, t_{2}}, \mathbb{R}^{n}\right)$. In addition, we have $u_{\delta} \geq \psi$, and the estimates from (3.7) and (3.8) persist in the limit because the upper bounds $C_{1}$ and $C_{2}$ are uniform with respect to $\varepsilon$ and $\gamma$. For the details, we refer to [7, Prop. 7.3].

Now, we are ready to show the existence of a strong solution to the obstacle problem.

Lemma 3.5. Suppose that the conditions (2.6)-(2.9) hold. Then, there exists a strong solution $u$ to the obstacle problem (2.5), which satisfies $u^{\frac{m+1}{2}} \in$ $L_{\mathrm{loc}}^{2}\left(0, T ; H_{\mathrm{loc}}^{1}(\Omega)\right)$.

Proof. By Lemma 3.4, there exists a weak solution $u_{\delta}$ to the penalized porous medium equation. From [11, Thm. 1.2], we know that the functions $u_{\delta}$ are locally Hölder continuous with a quantitative estimate which is uniform in $\delta$. Therefore, there is a function $u$ such that $u_{\delta} \rightarrow u$ locally uniformly in $\Omega_{T}$ 
as $\delta \rightarrow 0$. As the energy estimates in (3.5) and (3.6) are independent of $\delta$, we find a (not relabeled) subsequence $u_{\delta}$ such that $\nabla u_{\delta}^{m} \rightarrow \nabla u^{m}$ weakly in $L^{2}\left(\Omega_{T}, \mathbb{R}^{n}\right), u_{\delta} \stackrel{*}{\rightarrow} u$ weakly-* in $L^{\infty}\left(0, T ; L^{m+1}(\Omega)\right), \partial_{t} u_{\delta} \rightarrow \partial_{t} u$ weakly in $L^{2}\left(0, T ; H^{-1}(\Omega)\right)$, and $\nabla u_{\delta}^{\frac{m+1}{2}} \rightarrow \nabla u^{\frac{m+1}{2}}$ weakly in $L^{2}\left(U_{t_{1}, t_{2}}, \mathbb{R}^{n}\right)$, and, by the lower semicontinuity of the $L^{2}$-norm, we obtain

$$
\left\|\nabla u^{m}\right\|_{L^{2}\left(\Omega_{T}, \mathbb{R}^{n}\right)} \leq \liminf _{\delta \rightarrow 0+}\left\|\nabla u_{\delta}^{m}\right\|_{L^{2}\left(\Omega_{T}, \mathbb{R}^{n}\right)} \leq C_{1}
$$

and

$$
\left\|\nabla u^{\frac{m+1}{2}}\right\|_{L^{2}\left(U_{t_{1}, t_{2}}, \mathbb{R}^{n}\right)} \leq \liminf _{\delta \rightarrow 0+}\left\|\nabla u_{\delta}^{\frac{m+1}{2}}\right\|_{L^{2}\left(U_{t_{1}, t_{2}}, \mathbb{R}^{n}\right)} \leq C_{2}
$$

for any $U \Subset \Omega$ and $0<t_{1}<t_{2}<T$ with constants $C_{1}$ and $C_{2}$ as in (3.5) and (3.6). The fact that $u$ satisfies the variational inequality (2.11) follows from a calculation with the test function $\varphi=\alpha \eta\left(v^{m}-u_{\delta}^{m}+\delta \eta_{\delta}\right)$ in the weak formulation (3.2) of the penalized porous medium equation. Here, $\alpha$ and $\eta$ are cut-off functions in time and space, respectively, as in the definition of local strong solutions to the obstacle problem (see [7, Def. 2.1]), and $\eta_{\delta} \in C_{0}^{\infty}(\Omega)$ is a cut-off function such that

$$
\left\{\begin{array}{l}
\eta_{\delta}=1 \quad \text { in }\{x \in \Omega: \operatorname{dist}(x, \partial \Omega) \geq \delta\} \\
\left|\nabla \eta_{\delta}\right| \leq \frac{C}{\delta}
\end{array}\right.
$$

This shows that $u$ locally solves the obstacle problem and since it attains the correct boundary and initial values, it is also a strong solution to (2.5) by [7, Lemma 3.5]. For the details, we refer to [7, Section 8].

\section{Gradient estimates for Weak solutions}

As $\Psi$ is not bounded in general, strong solutions to the obstacle problem might not exist. Hence, we turn our attention to weak solutions. From now on, we will assume (2.6)-(2.8), but drop the condition (2.9). Our aim in this section is to prove the gradient estimate (4.2) for weak solutions by approximating them by strong solutions. Note that, in order to pass to the limit, the energy estimates from Section 3 do not suffice because they depend on $\Psi$.

We start with the following estimate, which can be found in [7, Lemma 9.1].

Lemma 4.1. Let $u$ be a weak solution to the obstacle problem (2.5). Then, we have

$$
\sup _{t \in[0, T]} \int_{\Omega} u(\cdot, t)^{m+1} d x+\iint_{\Omega_{T}}\left(u^{2 m}+\left|\nabla u^{m}\right|^{2}\right) d x d t \leq C \widetilde{A} .
$$

Here, $C$ is a constant depending on $n, m, \operatorname{diam}(\Omega)$ and $T$, and $\widetilde{A}$ is defined as

$$
\begin{aligned}
\widetilde{A}= & \sup _{t \in[0, T]} \int_{\Omega} g(\cdot, t)^{m+1} d x+\int_{\Omega} u_{0}^{m+1} d x \\
& +\iint_{\Omega_{T}}\left(g^{2 m}+\left|\nabla g^{m}\right|^{2}+\left|\partial_{t} g^{m}\right|^{\frac{m+1}{m}}\right) d x d t .
\end{aligned}
$$


Next, in order to control $\nabla u^{\frac{m+1}{2}}$, we recall that the strong solutions to the obstacle problem constructed in Lemma 3.5 are also weak supersolutions to the porous medium equation (see [7, Thm. 2.6]). Thus, the energy estimates for weak supersolutions are at our disposal. Let $U \Subset \Omega$ and $0<t_{1}<t_{2}<$ $T$. Then, provided that $u \leq M$ for some constant $M>0$, we may apply Lemma 2.3 with a suitable cut-off function $\zeta \in C_{0}^{\infty}\left(\Omega_{T}\right)$ to get

$$
\begin{aligned}
& \iint_{U_{t_{1}, t_{2}}}\left|\nabla u^{\frac{m+1}{2}}\right|^{2} d x d t \\
& \quad \leq C \iint_{\Omega_{T}}\left((M-u)^{2} \zeta\left|\partial_{t} \zeta\right|+u^{m-1}(M-u)^{2}|\nabla \zeta|^{2}\right) d x d t \leq C,
\end{aligned}
$$

where $C$ depends on $M, m,\left|\Omega_{T}\right|$ and $\operatorname{dist}\left(U_{t_{1}, t_{2}}, \partial \Omega_{T}\right)$. Here, $\left|\Omega_{T}\right|$ denotes the $(n+1)$-dimensional Lebesgue measure of the set $\Omega_{T}$.

We proceed to prove the existence of weak solutions to the obstacle problem whose gradients $\nabla u^{\frac{m+1}{2}}$ are locally bounded in $L^{2}\left(\Omega_{T}, \mathbb{R}^{n}\right)$. For the existence proof, we reproduce the key ideas from [7, Section 9] whereas our contribution is establishing the gradient estimate.

Lemma 4.2. Suppose that the conditions (2.6)-(2.8) hold. Then, there exists a weak solution to the obstacle problem (2.5), which satisfies $u^{\frac{m+1}{2}} \in$ $L_{\mathrm{loc}}^{2}\left(0, T ; H_{\mathrm{loc}}^{1}(\Omega)\right)$. Moreover, we have the estimate

$$
\left\|\nabla u^{\frac{m+1}{2}}\right\|_{L^{2}\left(U_{\left.t_{1}, t_{2}, \mathbb{R}^{n}\right)}\right.} \leq C
$$

for any $U \Subset \Omega$ and any $0<t_{1}<t_{2}<T$. Here, $C$ is a constant depending on $M, m,\left|\Omega_{T}\right|$ and $\operatorname{dist}\left(U_{t_{1}, t_{2}}, \partial \Omega_{T}\right)$, where the constant $M>0$ denotes the upper bounds for $\psi, g$ and $u_{0}$ from (2.6). Finally, $u$ is also a weak supersolution to the porous medium equation in $\Omega_{T}$, and if the obstacle $\psi$ is additionally Hölder continuous, then, $u$ is a weak solution to the porous medium equation in the set $\left\{(x, t) \in \Omega_{T}: u(x, t)>\psi(x, t)\right\}$.

Proof. Let $U \Subset \Omega$ and $0<t_{1}<t_{2}<T$ be fixed. We approximate the obstacle $\psi$ by a sequence of uniformly bounded obstacles $\left(\psi_{i}\right)_{i \in \mathbb{N}}$ satisfying

$$
\left\{\begin{array}{l}
\partial_{t} \psi_{i}-\Delta \psi_{i}^{m} \in L^{\infty}\left(\Omega_{T}\right), \\
\psi_{i}^{m} \rightarrow \psi^{m} \text { strongly in } L^{2}\left(0, T ; H^{1}(\Omega)\right), \\
\partial_{t} \psi_{i}^{m} \rightarrow \partial_{t} \psi^{m} \text { strongly in } L^{\frac{m+1}{m}}\left(\Omega_{T}\right), \\
\psi_{i}^{m}(\cdot, 0) \rightarrow \psi^{m}(\cdot, 0) \text { weakly in } H^{1}(\Omega) .
\end{array}\right.
$$

Note that, unlike in [7], it is not necessary to approximate $g$ and $u_{0}$ because of our boundedness assumption (2.6). By Lemma 3.5, the obstacle problem with $\psi_{i}$ as an obstacle has a strong solution $u_{i} \in C^{0}\left([0, T] ; L^{m+1}(\Omega)\right)$ satisfying $u_{i}^{m} \in g^{m}+L^{2}\left(0, T ; H_{0}^{1}(\Omega)\right)$ and $u_{i}^{\frac{m+1}{2}} \in L^{2}\left(t_{1}, t_{2} ; H^{1}(U)\right)$. We argue that the functions $u_{i}$ are uniformly bounded with respect to $i$ by a constant that can be determined in terms of $M$. First, since $\psi_{i}$ is uniformly bounded, we know that in the contact set $\left\{u_{i}=\psi_{i}\right\}$, also $u_{i}$ is uniformly bounded. Moreover, 
outside the contact set, $u_{i}$ is a weak solution to the porous medium equation by [7, Thm. 2.6]. Thus, the comparison principle from [3, Thm. 3.1] applied to $u_{i}$ and the upper bound for $\max \left\{\psi_{i}, g, u_{0}\right\}$ yields that $u_{i}$ is uniformly bounded in $\Omega_{T}$. Hence, by Lemma 4.1 and the Caccioppoli estimate (4.1), $u_{i}$ satisfies the estimates

$$
\sup _{t \in[0, T]} \int_{\Omega} u_{i}(\cdot, t)^{m+1} d x+\left\|u_{i}^{m}\right\|_{L^{2}\left(\Omega_{T}\right)}+\left\|\nabla u_{i}^{m}\right\|_{L^{2}\left(\Omega_{T}, \mathbb{R}^{n}\right)} \leq C_{1}
$$

and

$$
\left\|\nabla u_{i}^{\frac{m+1}{2}}\right\|_{L^{2}\left(U_{\left.t_{1}, t_{2}, \mathbb{R}^{n}\right)}\right.} \leq C_{2},
$$

where $C_{1}=C_{1}(n, m, \operatorname{diam}(\Omega), T, \widetilde{A})$ and $C_{2}=C_{2}\left(m, U, t_{1}, t_{2}, \Omega_{T}, M\right)$ are constants independent of $i$. Thus, there exist (not relabeled) subsequences $u_{i}^{m}$ and $u_{i}^{\frac{m+1}{2}}$ which are weakly convergent in $L_{\text {loc }}^{2}\left(0, T ; H_{\text {loc }}^{1}(\Omega)\right)$. In order to identify the limits, we will establish that $u_{i}^{m} \rightarrow u^{m}$ strongly in $L_{\mathrm{loc}}^{2}\left(\Omega_{T}\right)$. For that purpose, we introduce the time regularized functions

$$
w_{i, h}^{m}=\llbracket u_{i}^{m} \rrbracket_{h}-\llbracket \psi_{i}^{m} \rrbracket_{h}+\psi_{i}^{m} \quad \text { and } \quad w_{h}^{m}=\llbracket u^{m} \rrbracket_{h}-\llbracket \psi^{m} \rrbracket_{h}+\psi^{m},
$$

where we choose $v_{0}=u_{0}^{m}$ in $(2.2)$ to define $\llbracket u_{i}^{m} \rrbracket_{h}$, and analogous choices determine the other mollifications. The first step is showing that $u_{i} \rightarrow u$ in $L^{m+1}\left(U_{T}^{\varrho}\right)$, where $U_{T}^{\varrho}=B\left(x_{0}, \varrho\right) \times(0, T)$ with a ball $B\left(x_{0}, \varrho\right) \Subset \Omega$. By the triangular inequality, we have

$$
\begin{aligned}
& \left\|u_{i}-u\right\|_{L^{m+1}\left(U_{T}^{\rho}\right)} \\
& \quad \leq\left\|u_{i}-w_{i, h}\right\|_{L^{m+1}\left(U_{T}^{\rho}\right)}+\left\|w_{i, h}-w_{h}\right\|_{L^{m+1}\left(U_{T}^{\varrho}\right)}+\left\|w_{h}-u\right\|_{L^{m+1}\left(U_{T}^{\varrho}\right)} \\
& \quad=\mathrm{I}+\mathrm{II}+\mathrm{III} .
\end{aligned}
$$

We estimate the first term by $\left[7\right.$, ineq. (9.17)] to get $\mathrm{I} \leq C h^{\frac{1}{m+1}}$ for all $i \in \mathbb{N}$ and $h>0$ with a constant $C$ independent of $i$ and $h$. After that, we use the convergence $w_{i, h} \rightarrow w_{h}$ in $L^{2 m}\left(U_{T}^{\varrho}\right)$ from [7, eq. (9.13)] to find that II $\rightarrow 0$ as $i \rightarrow \infty$ for any $h>0$. Finally, the properties of the mollification (see [16, Lemma 2.2]) guarantee that III $\rightarrow 0$ as $h \rightarrow 0$.

Therefore, we conclude that $u_{i} \rightarrow u$ strongly in $L^{m+1}\left(U_{T}^{\varrho}\right)$, and an elementary computation shows that $u_{i}^{m} \rightarrow u^{m}$ strongly in $L^{\frac{m+1}{m}}\left(U_{T}^{\varrho}\right)$. Then, by applying Sobolev's inequality from Lemma 2.7 to $v=u_{i}^{m}$ with $p=2$ and $r=\frac{m+1}{m}$, we get

$$
\begin{aligned}
& \iint_{U_{T}^{\varrho}} u_{i}^{q} d x d t \\
& \quad \leq C \iint_{U_{T}^{\varrho}}\left(\left|\frac{u_{i}}{\varrho}\right|^{2 m}+\left|\nabla u_{i}^{m}\right|^{2}\right) d x d t\left(\sup _{t \in[0, T]} \int_{B\left(x_{0}, \varrho\right)} u_{i}(\cdot, t)^{m+1} d x\right)^{2 / n}
\end{aligned}
$$

with $q=2\left(m+\frac{m+1}{n}\right)>2 m$ and a constant $C$ independent of $i$. By the energy estimates (4.3) for $u_{i}$, the right-hand side is uniformly bounded with respect to $i$. Consequently, interpolation tells us $u_{i}^{m} \rightarrow u^{m}$ strongly in $L_{\text {loc }}^{2}\left(\Omega_{T}\right)$. Now, 
we can argue as in the proof of [7, Thm. 2.7] to conclude that $u$ is a weak solution to the obstacle problem in $\Omega_{T}$ and a weak supersolution to the porous medium equation in $\Omega_{T}$. Since the bound for $\nabla u_{i}^{\frac{m+1}{2}}$ from (4.4) is uniform with respect to $i$, it persists in the limit.

At this point, it remains to prove that $u$ is a weak solution to the porous medium equation in the set $\left\{z \in \Omega_{T}: u(z)>\psi(z)\right\}$ provided that the obstacle $\psi$ is Hölder continuous. To this aim, we first observe that, following the proof of [7, Thm. 2.7], it turns out that $u$ is also a local weak solution to the obstacle problem in the sense of [7, Def. 2.1]. Since we assumed that $\psi$ is Hölder continuous, we may apply [8, Thm. 1.1] to conclude that $u$ is locally Hölder continuous. Moreover, we know that $u$ is a weak solution to the obstacle problem on any subcylinder $Q \subset \Omega_{T}$. In the following, we consider a cylinder $Q=U \times\left[t_{1}, t_{2}\right) \Subset\left\{z \in \Omega_{T}: u(z)>\psi(z)\right\}$. Our aim is to prove that $u$ is a weak solution to the porous medium equation in $Q$ with initial datum $u_{0}=u\left(\cdot, t_{1}\right)$. Since $u$ and $\psi$ are continuous on $\bar{Q}$, there exists $\varepsilon>0$ such that $u^{m} \geq \psi^{m}+2 \varepsilon$ in $Q$. We construct the mollification in time $\llbracket u^{m} \rrbracket_{h}$ according to (2.2) subordinate to the cylinder $Q$ with initial values $v_{0}=u^{m}\left(\cdot, t_{1}\right)$. By similar arguments as in the proof of $\left[6\right.$, Lemma B.2(i)], we can show that $\llbracket u^{m} \rrbracket_{h} \rightarrow u^{m}$ uniformly in $Q$. Therefore, there exists $\tilde{h}>0$ such that $\left\|\llbracket u^{m} \rrbracket_{h}-u^{m}\right\|_{L^{\infty}(Q)} \leq \varepsilon$ for any $h \in(0, \tilde{h}]$ so that $\llbracket u^{m} \rrbracket_{h} \geq \psi^{m}+\varepsilon$ in $Q$. We now consider a function $\varphi \in C_{0}^{\infty}(Q)$ and prove that $(2.1)$ also holds for this test function. Without loss of generality, we may assume that $\inf _{Q} \varphi<0$ since otherwise there is nothing to prove. In the variational inequality $(2.13)$ on $Q$, we choose as comparison function

$$
v^{m}=\llbracket u^{m} \rrbracket_{h}+\tilde{\varepsilon} \varphi,
$$

where

$$
0<\tilde{\varepsilon}<\frac{\varepsilon}{-\inf _{Q} \varphi} .
$$

With this choice, we have that $v \geq \psi$ on $Q$ and hence, $v$ is admissible in (2.13). Therefore, we obtain

$$
\begin{aligned}
& \left\langle\left\langle\partial_{t} u, \alpha\left(\llbracket u^{m} \rrbracket_{h}-u^{m}+\tilde{\varepsilon} \varphi\right)\right\rangle_{u\left(\cdot, t_{1}\right)}\right. \\
& \quad+\iint_{Q} \alpha \nabla u^{m} \cdot \nabla\left(\llbracket u^{m} \rrbracket_{h}-u^{m}+\tilde{\varepsilon} \varphi\right) d x d t \geq 0
\end{aligned}
$$

for all non-negative cut-off functions $\alpha \in W^{1, \infty}\left(\left[t_{1}, t_{2}\right]\right)$ with $\alpha\left(t_{2}\right)=0$. Here, we choose

$$
\alpha(t):= \begin{cases}1 & \text { for } t \in\left[t_{1}, t_{2}-\delta\right], \\ \frac{t_{2}-t}{\delta} & \text { for } t \in\left(t_{2}-\delta, t_{2}\right]\end{cases}
$$

with $\delta \in\left(0, t_{2}-t_{1}\right)$. Taking into account

$$
-\iint_{Q} \alpha u \partial_{t} \llbracket u^{m} \rrbracket_{h} d x d t
$$




$$
\begin{aligned}
& =-\iint_{Q} \alpha \llbracket u^{m} \rrbracket_{h}^{\frac{1}{m}} \partial_{t} \llbracket u^{m} \rrbracket_{h} d x d t-\iint_{Q} \alpha\left[u-\llbracket u^{m} \rrbracket_{h}^{\frac{1}{m}}\right] \partial_{t} \llbracket u^{m} \rrbracket_{h} d x d t \\
& =-\frac{m}{m+1} \iint_{Q} \alpha \partial_{t} \llbracket u^{m} \rrbracket_{h}^{\frac{m+1}{m}} d x d t-\frac{1}{h} \iint_{Q} \alpha\left[u-\llbracket u^{m} \rrbracket_{h}^{\frac{1}{m}}\right]\left[u^{m}-\llbracket u^{m} \rrbracket_{h}\right] d x d t \\
& \leq \frac{m}{m+1} \iint_{Q} \alpha^{\prime} \llbracket u^{m} \rrbracket_{h}^{\frac{m+1}{m}} d x d t+\frac{m}{m+1} \int_{U} u^{m+1}\left(\cdot, t_{1}\right) d x
\end{aligned}
$$

where we used (2.4), we may compute for the first integral on the left-hand side of (4.5) that

$$
\begin{aligned}
\left\langle\left\langle\partial_{t} u, \alpha\right.\right. & \left.\left.\left(\llbracket u^{m} \rrbracket_{h}-u^{m}+\delta \varphi\right)\right\rangle\right\rangle_{u\left(\cdot, t_{1}\right)} \\
\leq & \iint_{Q} \alpha^{\prime}\left(\frac{1}{m+1} u^{m+1}+\frac{m}{m+1} \llbracket u^{m} \rrbracket_{h}^{\frac{m+1}{m}}-u \llbracket u^{m} \rrbracket_{h}-\tilde{\varepsilon} u \varphi\right) d x d t \\
& -\tilde{\varepsilon} \iint_{Q} \alpha u \partial_{t} \varphi d x d t .
\end{aligned}
$$

Since $\varphi\left(\cdot, t_{1}\right)=0$ and $\llbracket u^{m} \rrbracket_{h} \rightarrow u^{m}$ in $L^{\frac{m+1}{m}}(Q)$, the terms on right-hand side converge to

$$
-\tilde{\varepsilon} \iint_{Q} u \partial_{t} \varphi d x d t
$$

as $h, \delta \downarrow 0$. Moreover, the second integral on the left-hand side of (4.5) tends to

$$
\tilde{\varepsilon} \iint_{Q} \nabla u^{m} \cdot \nabla \varphi d x d t
$$

in the limit $h, \delta \downarrow 0$. Therefore, we have shown that the inequality (2.1) holds for each $\varphi \in C_{0}^{\infty}\left(\Omega_{T}\right)$ without any assumption on the sign of $\varphi$. This implies that $u$ is a weak solution to the porous medium equation in $Q$. Since $Q$ was an arbitrary cylinder in the set $\left\{z \in \Omega_{T}: u(z)>\psi(z)\right\}$, Lemma 4.2 is proven.

\section{Proof of the MAin Results}

In this section, we will prove Theorem 1.1 and Theorem 1.2. We begin with the proof of the former one. The approximation by solutions to the obstacle problem follows the ideas of [17, Thm. 1.3], and the novelty in our paper is establishing gradient estimates which ensure that the regularity property (1.2) is valid.

Proof of Theorem 1.1. Since $u$ is locally bounded and lower semicontinuous by assumption, there exists a sequence of locally uniformly bounded functions $\psi_{i} \in C^{\infty}\left(\Omega_{T}\right)$ such that

$$
\psi_{i}<\psi_{i+1} \text { for any } i \in \mathbb{N} \text { and } \lim _{i \rightarrow \infty} \psi_{i}(x, t)=u(x, t) \text { for a. e. }(x, t) \in \Omega_{T} \text {. }
$$

Without loss of generality, we may consider sets $V_{\tau_{1}, \tau_{2}} \Subset U_{t_{1}, t_{2}} \Subset \Omega_{T}$. Then, Lemma 4.2 ensures that, for each $i$, there exists a weak solution $u_{i}$ to the 
obstacle problem for the porous medium equation in $U_{t_{1}, t_{2}}$ with obstacle and initial and lateral boundary data $\psi_{i}$, i. e. $u_{i}$ satisfies

$$
\left\{\begin{array}{l}
u_{i}^{m} \in \psi_{i}^{m}+L^{2}\left(t_{1}, t_{2} ; H_{0}^{1}(U)\right) \\
u_{i} \geq \psi_{i} \text { a.e. in } U_{t_{1}, t_{2}}
\end{array}\right.
$$

and

$$
\left\langle\left\langle\partial_{t} u_{i}, \alpha\left(v^{m}-u_{i}^{m}\right)\right\rangle\right\rangle_{\psi_{i}\left(\cdot, t_{1}\right)}+\iint_{U_{t_{1}, t_{2}}} \alpha \nabla u_{i}^{m} \cdot \nabla\left(v^{m}-u_{i}^{m}\right) d x d t \geq 0
$$

for all comparison maps $v \in \psi_{i}^{m}+L^{2}\left(t_{1}, t_{2} ; H_{0}^{1}(U)\right)$ with $v \geq \psi_{i}$ a.e. in $U_{t_{1}, t_{2}}$ and $\partial_{t} v^{m} \in L^{\frac{m+1}{m}}\left(U_{t_{1}, t_{2}}\right)$, and for all non-negative cut-off functions $\alpha \in W^{1, \infty}\left(\left[t_{1}, t_{2}\right]\right)$ with $\alpha\left(t_{2}\right)=0$. By Lemma 4.2, for each $i, u_{i}$ is a weak sopersolution to the porous medium equation in $U_{t_{1}, t_{2}}$ and a weak solution to the porous medium equation in the set $\left\{(x, t) \in U_{t_{1}, t_{2}}: u_{i}(x, t)>\psi_{i}(x, t)\right\}$. Furthermore, by Lemma 4.1 and Lemma 4.2, the gradients satisfy

$$
\left\|\nabla u_{i}^{m}\right\|_{L^{2}\left(U_{t_{1}, t_{2}}, \mathbb{R}^{n}\right)} \leq C \quad \text { and } \quad\left\|\nabla u_{i}^{\frac{m+1}{2}}\right\|_{L^{2}\left(V_{\tau_{1}, \tau_{2}}, \mathbb{R}^{n}\right)} \leq C
$$

with a constant $C$ that is uniform with respect to $i$. On the other hand, by [8, Thm. 1.1] and Remark 2.6, $u_{i}$ is a locally continuous weak supersolution in $U_{t_{1}, t_{2}}$. In order to conclude that $u_{i} \rightarrow u$ in $L^{2}\left(V_{\tau_{1}, \tau_{2}}\right)$, we will show that

$$
u_{i} \leq u_{i+1} \leq u \text { a. e. in } V_{\tau_{1}, \tau_{2}} \text { for any } i .
$$

To this aim, we consider the sets

$$
K_{i}=\left\{(x, t) \in \overline{V_{\tau_{1}, \tau_{2}}}: u_{i}(x, t) \geq \psi_{i+1}(x, t)\right\} .
$$

Since $\psi_{i+1}>\psi_{i}$ and the functions $u_{i}$ and $\psi_{i}$ are continuous in $V_{\tau_{1}, \tau_{2}}$ for every $i$, the set $K_{i}$ is compact. If $K_{i}=\emptyset$, we have $u_{i}<\psi_{i+1}$ in $\overline{V_{\tau_{1}, \tau_{2}}}$, which implies $u_{i}<u_{i+1}$ and $u_{i}<u$. Therefore, it remains to consider the case $K_{i} \neq \emptyset$. Then, the distance

$$
d=\operatorname{dist}\left(K_{i},\left\{(x, t) \in \overline{V_{\tau_{1}, \tau_{2}}}: u_{i}(x, t)=\psi_{i}(x, t)\right\}\right)
$$

is positive. As $K_{i}$ is compact, there exists a finite number $N$ such that $K_{i}$ can be covered with $N$ dyadic cubes $Q_{j}$ with diameter $\operatorname{diam}\left(Q_{j}\right)<d / 2$. Hence, we may define

$$
Q=\bigcup_{j=1}^{N} Q_{j} \subset\left\{(x, t) \in \overline{V_{\tau_{1}, \tau_{2}}}: u_{i}(x, t)>\psi_{i}(x, t)\right\}
$$

and conclude that $u_{i}$ is a weak solution to the porous medium equation in $Q$ because the contact set $\left\{u_{i}=\psi_{i}\right\}$ does not intersect $Q$. By construction, we have $u_{i} \leq \psi_{i+1}<u$ on $\partial_{p} Q$, where $\partial_{p}$ denotes the natural generalization of the parabolic boundary for finite unions of cylinders (see [3, Section 3] for the exact definition). Since the comparison principle from Def. 2.2 (3) can be applied to $u_{i}$ and $u$ in such a set (see the argument in [19, Rem. 3.4]), we deduce that $u_{i} \leq u$ in $Q \supset K_{i}$. In addition, the inequality $u_{i}<\psi_{i+1}<u$ holds 
in $\overline{V_{\tau_{1}, \tau_{2}}} \backslash K_{i}$ by the definition of the set $K_{i}$. As $\varepsilon>0$ was arbitrary, we infer that $u_{i} \leq u$ in $V_{\tau_{1}, \tau_{2}}$.

On the other hand, we have $u_{i} \leq \psi_{i+1} \leq u_{i+1}$ on $\partial_{p} Q$. Since $u_{i}$ is a weak solution to the porous medium equation and $u_{i+1}$ is a weak supersolution in $Q$, we may use the comparison principle from [3, Thm. 3.1] to find that $u_{i} \leq u_{i+1}$ in $Q \supset K_{i}$. Again, in the set $\overline{V_{\tau_{1}, \tau_{2}}} \backslash K_{i}$, the inequality $u_{i}<\psi_{i+1} \leq u_{i+1}$ holds by the definition of $K_{i}$. Thus, we obtain that $u_{i} \leq u_{i+1}$ in $V_{\tau_{1}, \tau_{2}}$.

Collecting the facts, we see that

$$
\psi_{i} \leq u_{i} \leq u_{i+1} \leq u \text { a. e. in } V_{\tau_{1}, \tau_{2}} \text { for any } i \text { and } \psi_{i} \rightarrow u
$$

From this and the dominated convergence theorem, we know $u_{i} \rightarrow u$ in $L^{2}\left(V_{\tau_{1}, \tau_{2}}\right)$. It remains to show that $u$ is a weak supersolution satisfying (1.2). By (5.1) and weak compactness, we deduce the existence of the gradients $\nabla u^{m}$ and $\nabla u^{\frac{m+1}{2}}$ as well as the convergences

$$
\begin{aligned}
& \nabla u_{i}^{m} \neg \nabla u^{m} \text { weakly in } L^{2}\left(U_{t_{1}, t_{2}}, \mathbb{R}^{n}\right) \text { and } \\
& \nabla u_{i}^{\frac{m+1}{2}} \neg \nabla u^{\frac{m+1}{2}} \text { weakly in } L^{2}\left(V_{\tau_{1}, \tau_{2}}, \mathbb{R}^{n}\right)
\end{aligned}
$$

for (not relabeled) subsequences of $\nabla u_{i}^{m}$ and $\nabla u_{i}^{\frac{m+1}{2}}$. Since $V_{\tau_{1}, \tau_{2}} \Subset \Omega_{T}$ was arbitrary, we conclude that $\nabla u^{m} \in L_{\text {loc }}^{2}\left(\Omega, \mathbb{R}^{n}\right)$ and $\nabla u^{\frac{m+1}{2}} \in L_{\text {loc }}^{2}\left(\Omega, \mathbb{R}^{n}\right)$. Finally, due to the convergences $u_{i} \rightarrow u$ in $L_{\text {loc }}^{2}\left(\Omega_{T}\right)$ and $\nabla u_{i}^{m} \rightarrow \nabla u^{m}$ weakly in $L_{\text {loc }}^{2}\left(\Omega_{T}, \mathbb{R}^{n}\right)$, we may pass to the limit $i \rightarrow \infty$ in

$$
\iint_{\Omega_{T}}\left(-u_{i} \partial_{t} \varphi+\nabla u_{i}^{m} \cdot \nabla \varphi\right) d x d t \geq 0
$$

and conclude that $u$ satisfies (2.1). Moreover, using the time mollification (2.2) we can show by an argument similar to the proof of [7, Lemma 5.2] that $u \in C^{0}\left((0, T) ; L_{\text {loc }}^{m+1}(\Omega)\right)$. This ensures that $u$ is a weak supersolution to the porous medium equation and finishes the proof of Theorem 1.1.

As a consequence of Theorem 1.1, we may now prove Theorem 1.2.

Proof of Theorem 1.2. First, let $u$ satisfy (i). From [11, Thm. 1.2] and [9, Thm. 1.1], respectively, we know that $u$ has a locally continuous representative. Without loss of generality, we may assume that $u$ itself is locally continuous and, in particular, locally bounded. Furthermore, due to [21, Thm. 6.5], the comparison principle holds for $u$. Therefore, we conclude that $u$ is $m$-supercaloric, and hence, Theorem 1.1 ensures $u^{\frac{m+1}{2}} \in$ $L_{\text {loc }}^{2}\left(0, T ; H_{\text {loc }}^{1}(\Omega)\right)$. Moreover, the assumption $u \in C^{0}\left((0, T) ; L_{\text {loc }}^{m+1}(\Omega)\right)$ implies $u \in C^{0}\left((0, T) ; L_{\mathrm{loc}}^{2}(\Omega)\right)$ so that assertion (ii) is verified.

On the other hand, suppose that $u$ satisfies (ii). Since $u \in L_{\mathrm{loc}}^{m}\left(\Omega_{T}\right)$, we may apply [9, Thm. 1.1] to find that $u$ is locally bounded. In order to show the existence of $\nabla u^{m}$, we let $k \in \mathbb{N}$ and consider the truncations $u_{k}=\min \{u, k\}$. Since the mapping $[0, k] \ni s \mapsto s^{\frac{2 m}{m+1}}$ is Lipschitz continuous, we conclude that 
$u_{k}^{m}$ is weakly differentiable and

$$
\nabla u_{k}^{m}=\nabla\left[\left(u_{k}^{\frac{m+1}{2}}\right)^{\frac{2 m}{m+1}}\right]=\frac{2 m}{m+1} u_{k}^{\frac{m-1}{2}} \nabla u_{k}^{\frac{m+1}{2}}
$$

so that

$$
\iint_{U_{t_{1}, t_{2}}} u_{k}^{m} \partial_{x_{i}} \varphi d x d t=-\frac{2 m}{m+1} \iint_{U_{t_{1}, t_{2}}} u_{k}^{\frac{m-1}{2}} \partial_{x_{i}}\left(u_{k}^{\frac{m+1}{2}}\right) \varphi d x d t
$$

for any $i \in\{1, \ldots, n\}$, any $U_{t_{1}, t_{2}} \Subset \Omega_{T}$ and any $\varphi \in C_{0}^{\infty}\left(U_{t_{1}, t_{2}}\right)$. We recall that, by assumption, we have $u^{\frac{m+1}{2}} \in L_{\text {loc }}^{2}\left(0, T ; H_{\text {loc }}^{1}(\Omega)\right)$. Therefore, we can infer strong convergence of $u_{k}$ in $L^{m+1}\left(U_{t_{1}, t_{2}}\right)$ as well as weak subconvergence of $\nabla u_{k}^{\frac{m+1}{2}}$ in $L^{2}\left(U_{t_{1}, t_{2}}, \mathbb{R}^{n}\right)$. This allows us to pass to the limit $k \rightarrow \infty$ in the above equation concluding that the weak gradient of $u^{m}$ exists and is given by $\frac{2 m}{m+1} u^{\frac{m-1}{2}} \nabla u^{\frac{m+1}{2}}$. Consequently, the weak formulations from (i) and (ii) coincide. Moreover, due to the local boundedness of $u$, we obtain

$$
\begin{aligned}
\iint_{U_{t_{1}, t_{2}}}\left|\nabla u^{m}\right|^{2} d x d t & =\left(\frac{2 m}{m+1}\right)^{2} \iint_{U_{t_{1}, t_{2}}} u^{m-1}\left|\nabla u^{\frac{m+1}{2}}\right|^{2} d x d t \\
& \leq C \iint_{U_{t_{1}, t_{2}}}\left|\nabla u^{\frac{m+1}{2}}\right|^{2} d x d t<\infty,
\end{aligned}
$$

where the constant $C$ depends on $m$ and the upper bound for $u$ in $U_{t_{1}, t_{2}}$. This ensures that $\nabla u^{m} \in L^{2}\left(U_{t_{1}, t_{2}}, \mathbb{R}^{n}\right)$. Together with the fact that $u$ satisfies the weak formulation of the porous medium equation, this implies that $u \in$ $C^{0}\left(\left[t_{1}, t_{2}\right) ; L_{\text {loc }}^{m+1}(U)\right)$; cf. [7, Lemma 5.2]. Since $U_{t_{1}, t_{2}} \Subset \Omega_{T}$ was arbitrary, we deduce that (i) holds, which finishes the proof.

Alternative proof of Theorem 1.2, $(i) \Rightarrow($ ii $)$. Let $u$ satisfy (i). For $\varepsilon>0$, we let $g_{\varepsilon}(s):=\max \{\varepsilon, s\}=\max \left\{\varepsilon^{m}, s^{m}\right\}^{\frac{1}{m}}$ for any $s \geq 0$. Since the mapping $\mathbb{R} \ni \sigma \mapsto \max \left\{\varepsilon^{m}, \sigma\right\}^{\frac{1}{m}}$ is Lipschitz continuous, we know that $g_{\varepsilon}(u)=\max \left\{\varepsilon^{m}, u^{m}\right\}^{\frac{1}{m}}$ is weakly differentiable and

$$
\nabla g_{\varepsilon}(u)=\frac{1}{m} \chi_{\{u>\varepsilon\}} u^{1-m} \nabla u^{m} \in L_{\mathrm{loc}}^{2}\left(\Omega_{T}, \mathbb{R}^{n}\right)
$$

so that $g_{\varepsilon}(u) \in L_{\text {loc }}^{2}\left(0, T ; H_{\text {loc }}^{1}(\Omega)\right)$. Moreover, we recall the mollification in time defined in (2.2), where, throughout this proof, we choose $v_{0}=0$ as initial value. Similar to inequality (2.3) for weak supersolutions, we can derive the following regularized version of (1.3):

$$
\iint_{\Omega_{T}}\left(\partial_{t} \llbracket u \rrbracket_{h} \varphi+\nabla \llbracket u^{m} \rrbracket_{h} \cdot \nabla \varphi\right) d x d t=\frac{1}{h} \int_{\Omega} u(\cdot, 0) \int_{0}^{T} \varphi e^{-\frac{s}{h}} d s d x
$$

for any test function $\varphi \in L^{2}\left(0, T ; H_{0}^{1}(\Omega)\right)$. In (5.2), we insert $\varphi=\zeta^{2} g_{\varepsilon}(u)$, where $\zeta \in C_{0}^{\infty}\left(\Omega_{T}\right)$ is a smooth cut-off function with $0 \leq \zeta \leq 1$. We first treat the evolutionary integral. Using formula (2.4) for the time derivative of the mollification, integrating by parts, and then, passing to the limit $h \rightarrow 0$, we 
find

$$
\begin{aligned}
& \iint_{\Omega_{T}} \zeta^{2} \partial_{t} \llbracket u \rrbracket_{h} g_{\varepsilon}(u) d x d t \\
& \quad=\iint_{\Omega_{T}} \zeta^{2} \partial_{t} \llbracket u \rrbracket_{h} g_{\varepsilon}\left(\llbracket u \rrbracket_{h}\right) d x d t+\iint_{\Omega_{T}} \zeta^{2} \partial_{t} \llbracket u \rrbracket_{h}\left(g_{\varepsilon}(u)-g_{\varepsilon}\left(\llbracket u \rrbracket_{h}\right)\right) d x d t \\
& \quad \geq \iint_{\Omega_{T}} \zeta^{2} \partial_{t}\left(\int_{0}^{\llbracket u \rrbracket_{h}} g_{\varepsilon}(s) d s\right) d x d t=-2 \iint_{\Omega_{T}} \zeta \partial_{t} \zeta \int_{0}^{\llbracket u \rrbracket_{h}} g_{\varepsilon}(s) d s d x d t \\
& \quad=-2 \iint_{\Omega_{T}} \zeta \partial_{t} \zeta G_{\varepsilon}\left(\llbracket u \rrbracket_{h}\right) d x d t \rightarrow-2 \iint_{\Omega_{T}} \zeta \partial_{t} \zeta G_{\varepsilon}(u) d x d t,
\end{aligned}
$$

where

$$
G_{\varepsilon}(s):= \begin{cases}\varepsilon s & \text { for } 0 \leq s \leq \varepsilon \\ \frac{1}{2}\left(\varepsilon^{2}+s^{2}\right) & \text { for } s>\varepsilon .\end{cases}
$$

Note that the right-hand side term in (5.2) vanishes as $h \rightarrow 0$, and, for the diffusion term, we get in the limit $h \rightarrow 0$ that

$$
\begin{aligned}
& \iint_{\Omega_{T}} \nabla \llbracket u^{m} \rrbracket_{h} \cdot \nabla\left(\zeta^{2} g_{\varepsilon}(u)\right) d x d t \rightarrow \iint_{\Omega_{T}} \nabla u^{m} \cdot \nabla\left(\zeta^{2} g_{\varepsilon}(u)\right) d x d t \\
& \quad=2 \iint_{\Omega_{T}} \zeta g_{\varepsilon}(u) \nabla u^{m} \cdot \nabla \zeta d x d t+\iint_{\Omega_{T}} \zeta^{2} \nabla u^{m} \cdot \nabla g_{\varepsilon}(u) d x d t .
\end{aligned}
$$

For the first integral on the right-hand side, we compute

$$
\begin{aligned}
\left|\iint_{\Omega_{T}} \zeta g_{\varepsilon}(u) \nabla u^{m} \cdot \nabla \zeta d x d t\right| & \leq \iint_{\Omega_{T}} g_{\varepsilon}(u)\left|\nabla u^{m}\right||\nabla \zeta| d x d t \\
& \leq \iint_{\Omega_{T}}\left[|\nabla \zeta|^{2} g_{\varepsilon}(u)^{2}+\left|\nabla u^{m}\right|^{2}\right] d x d t
\end{aligned}
$$

while, for the second one, we find

$$
\begin{aligned}
\iint_{\Omega_{T}} \zeta^{2} \nabla u^{m} \cdot \nabla g_{\varepsilon}(u) d x d t & =\frac{1}{m} \iint_{\Omega_{T} \cap\{u>\varepsilon\}} \zeta^{2} u^{1-m} \nabla u^{m} \cdot \nabla u^{m} d x d t \\
& =\frac{4 m}{(m+1)^{2}} \iint_{\Omega_{T} \cap\{u>\varepsilon\}} \zeta^{2}\left|\nabla u^{\frac{m+1}{2}}\right|^{2} d x d t,
\end{aligned}
$$

where we have defined $\nabla u^{\frac{m+1}{2}}:=\frac{m+1}{2 m} \chi_{\{u>0\}} u^{\frac{1-m}{2}} \nabla u^{m}$. Combining the preceding computations, we see that

$$
\begin{aligned}
& \iint_{\Omega_{T} \cap\{u>\varepsilon\}} \zeta^{2}\left|\nabla u^{\frac{m+1}{2}}\right|^{2} d x d t \\
& \quad \leq \frac{(m+1)^{2}}{2 m} \iint_{\Omega_{T}}\left[\left|\partial_{t} \zeta\right| G_{\varepsilon}(u)+|\nabla \zeta|^{2} g_{\varepsilon}(u)^{2}+\left|\nabla u^{m}\right|^{2}\right] d x d t .
\end{aligned}
$$


Note that the right-hand side is bounded uniformly with respect to $\varepsilon$ and converges as $\varepsilon \downarrow 0$. Therefore, we obtain by Fatou's lemma that

$$
\begin{aligned}
& \iint_{\Omega_{T}} \zeta^{2}\left|\nabla u^{\frac{m+1}{2}}\right|^{2} d x d t \\
& \quad \leq \liminf _{\varepsilon \downarrow 0} \iint_{\Omega_{T} \cap\{u>\varepsilon\}} \zeta^{2}\left|\nabla u^{\frac{m+1}{2}}\right|^{2} d x d t \\
& \quad \leq \lim _{\varepsilon \downarrow 0} \frac{(m+1)^{2}}{2 m} \iint_{\Omega_{T}}\left[\left|\partial_{t} \zeta\right| G_{\varepsilon}(u)+|\nabla \zeta|^{2} g_{\varepsilon}(u)^{2}+\left|\nabla u^{m}\right|^{2}\right] d x d t \\
& \quad=\frac{(m+1)^{2}}{2 m} \iint_{\Omega_{T}}\left[\left(\frac{1}{2}\left|\partial_{t} \zeta\right|+|\nabla \zeta|^{2}\right) u^{2}+\left|\nabla u^{m}\right|^{2}\right] d x d t .
\end{aligned}
$$

This ensures that $\nabla u^{\frac{m+1}{2}} \in L_{\text {loc }}^{2}\left(\Omega_{T}, \mathbb{R}^{n}\right)$. It remains to show that $\nabla u^{\frac{m+1}{2}}$ is the weak derivative of $u^{\frac{m+1}{2}}$. To this aim, we consider $\varphi \in C_{0}^{\infty}\left(\Omega_{T}\right)$ and compute for $i \in\{1, \ldots, n\}$ that

$$
\begin{aligned}
\iint_{\Omega_{T}} u^{\frac{m+1}{2}} \partial_{x_{i}} \varphi d x d t & =\iint_{\Omega_{T}}\left(u^{m}\right)^{\frac{m+1}{2 m}} \partial_{x_{i}} \varphi d x d t \\
& =-\frac{m+1}{2 m} \iint_{\Omega_{T}} \chi_{\{u>0\}} u^{\frac{1-m}{2}} \partial_{x_{i}}\left(u^{m}\right) \varphi d x d t \\
& =-\iint_{\Omega_{T}} \nabla u^{\frac{m+1}{2}} \cdot e_{i} \varphi d x d t
\end{aligned}
$$

which proves the assertion that $\nabla u^{\frac{m+1}{2}}$ is the weak derivative of $u^{\frac{m+1}{2}}$, and hence $u^{\frac{m+1}{2}} \in L_{\mathrm{loc}}^{2}\left(0, T ; H_{\mathrm{loc}}^{1}(\Omega)\right)$. Moreover, the assumption $u \in$ $C^{0}\left((0, T) ; L_{\mathrm{loc}}^{m+1}(\Omega)\right)$ implies $u \in C^{0}\left((0, T) ; L_{\mathrm{loc}}^{2}(\Omega)\right)$. Finally, since $\nabla u^{m}=$ $\frac{m+1}{2 m} u^{\frac{m-1}{2}} \nabla u^{\frac{m+1}{2}}$ by the very definition of $\nabla u^{\frac{m+1}{2}}$, we can deduce (1.4) from (1.3) so that assertion (ii) is verified.

\section{REFERENCES}

[1] Daniele Andreucci. $L_{\mathrm{loc}}^{\infty}$-estimates for local solutions of degenerate parabolic equations. SIAM J. Math. Anal., 22(1):138-145, 1991.

[2] Benny Avelin and Teemu Lukkari. Lower semicontinuity of weak supersolutions to the porous medium equation. Proc. Amer. Math. Soc., 143(8):3475-3486, 2015.

[3] Benny Avelin and Teemu Lukkari. A comparison principle for the porous medium equation and its consequences. Rev. Mat. Iberoam., 33(2):573-594, 2017.

[4] Verena Bögelein, Frank Duzaar, and Ugo Gianazza. Porous medium type equations with measure data and potential estimates. SIAM J. Math. Anal., 45(6):3283-3330, 2013.

[5] Verena Bögelein, Frank Duzaar, Riikka Korte, and Christoph Scheven. The higher integrability of weak solutions of porous medium systems.

[6] Verena Bögelein, Frank Duzaar, and Paolo Marcellini. Parabolic systems with $p, q$ growth: a variational approach. Arch. Ration. Mech. Anal., 210(1):219-267, 2013.

[7] Verena Bögelein, Teemu Lukkari, and Christoph Scheven. The obstacle problem for the porous medium equation. Math. Ann., 363(1-2):455-499, 2015.

[8] Verena Bögelein, Teemu Lukkari, and Christoph Scheven. Hölder regularity for degenerate parabolic obstacle problems. Ark. Mat., 55:1-39, 2017. 
[9] Björn E. J. Dahlberg and Carlos E. Kenig. Weak solutions of the porous medium equation. Trans. Amer. Math. Soc., 336(2):711-725, 1993.

[10] Emmanuele DiBenedetto. Degenerate parabolic equations. Universitext. SpringerVerlag, New York, 1993.

[11] Emmanuele DiBenedetto and Avner Friedman. Hölder estimates for nonlinear degenerate parabolic systems. J. Reine Angew. Math., 357:1-22, 1985.

[12] Emmanuele DiBenedetto, Ugo Gianazza, and Vincenzo Vespri. Harnack estimates for quasi-linear degenerate parabolic differential equations. Acta Math., 200:181-209, 2008.

[13] Emmanuele DiBenedetto, Ugo Gianazza, and Vincenzo Vespri. Harnack's inequality for degenerate and singular parabolic equations. Springer Monographs in Mathematics. Springer, New York, 2012.

[14] Ugo Gianazza and Sebastian Schwarzacher. Self-improving property of degenerate parabolic equations of porous medium-type. Available at https://arxiv.org/abs/1603.07241.

[15] Aleksandr Vasil'evich Ivanov, P. Z. Mkrtychyan, and Willi Yäger. Existence and uniqueness of a regular solution of the first initial-boundary value problem for a class of doubly nonlinear parabolic equations. Zap. Nauchn. Sem. S.-Peterburg. Otdel. Mat. Inst. Steklov. (POMI), 213(Kraev. Zadachi Mat. Fiz. Smezh. Voprosy Teor. Funktsiu. 25):4865, 224-225, 1994.

[16] Juha Kinnunen and Peter Lindqvist. Pointwise behaviour of semicontinuous supersolutions to a quasilinear parabolic equation. Ann. Mat. Pura Appl. (4), 185(3):411-435, 2006.

[17] Juha Kinnunen and Peter Lindqvist. Definition and properties of supersolutions to the porous medium equation. J. Reine Angew. Math., 618:135-168, 2008.

[18] Juha Kinnunen and Peter Lindqvist. Unbounded supersolutions of some quasilinear parabolic equations: a dichotomy. Nonlinear Anal., 131:229-242, 2016.

[19] Pekka Lehtelä and Teemu Lukkari. The equivalence of weak and very weak supersolutions to the porous medium equation. Tohoku Math. J., to appear, 2018. Available at https://arxiv.org/abs/1603.03641.

[20] Jacques-Louis Lions. Quelques méthodes de résolution des problèmes aux limites non linéaires. Dunod; Gauthier-Villars, Paris, 1969.

[21] Juan Luis Vázquez. The porous medium equation. Oxford Mathematical Monographs. The Clarendon Press, Oxford University Press, Oxford, 2007. Mathematical theory.

Verena Bögelein, Fachbereich Mathematik, Universität Salzburg, HellBrunner Str. 34, 5020 SAlzburg, Austria

E-mail address: verena.boegelein@sbg.ac.at

Pekka Lehtelä, Aalto University, Department of Mathematics and Systems Analysis, P.O. Box 11100, FI-00076 Aalto, Finland

E-mail address: pekka.lehtela@aalto.fi

Stefan Sturm, Fachbereich Mathematik, Universität Salzburg, HellbrunNER Str. 34, 5020 Salzburg, Austria

E-mail address: stefan.sturm@sbg.ac.at 